\title{
A brief review of WSN applications
}

\author{
Jingjing Zhao ${ }^{\text {ab,*, }}$ Meng Ge ${ }^{\text {a, }}$ Yun Yang ${ }^{\text {b }}$, Lifeng Zhang ${ }^{\text {a }}$ \\ ${ }^{a}$ Department of Electrical and Electronic Engineering, Kyushu Institute of Technology, Kitakyushu, Japan \\ ${ }^{\mathrm{b}}$ College of Information Engineering, Yangzhou University, Yangzhou, China
}

*q349430s@mail.kyutech.jp

\begin{abstract}
With the rapid technological developments of sensors, Wireless Sensor Networks (WSNs) have become the main technology for IoT ${ }^{(1)}$, which means Internet of Things. Nowadays, the wireless network is used more wildly in different places for its low-cost and easy-setup compared to the wired network, resulting in more and more applications of WSN in various fields. For studying of the WSN and applying it into my research, this paper briefly gives the concept of WSN and a review of WSN applications.
\end{abstract}

Keywords: WSN, WSN applications.

\section{Introduction}

A wireless network serves the same purpose as a wired one - to link a group of sensors, such as laptops to the Internet and to your business network and its applications. When you connect a laptop to a Wi-Fi hotspot at a cafe, hotel, airport lounge, or other public place, you're connecting to that business's wireless network ${ }^{(2)}$. As "wireless" doesn't require costly wiring, which means it's generally easier, faster and cheaper to set up, the wireless network is the best choice for sensor networks.

Today the wireless sensor networks is applied in more and more areas, such as environmental monitoring and protection, medical care, military monitoring and so on, so wireless sensor network has become an indispensable part for us to create a better life.

The section 2 in this paper introduces what the WSN is and its characteristics. Research on WSNs dates back to the early 1980s when the United States Defense Advanced Research Projects Agency (DARPA) carried out the distributed sensor networks (DSNs) program for the US military. At that time, the Advanced Research Projects Agency Network (ARPANET) had been in operation for a number of years, with about 200 hosts at universities and research institutes ${ }^{(3)}$.

The section 3 gives a review of WSN applications. There are numerous WSN applications, but ultimately they all fall into two categories: monitoring and tracking. Furthermore, applications vary depending on the environment where the sensors are deployed ${ }^{(4)}$.

\section{WSN (Wireless Sensor Networks)}

\subsection{Concept of WSN}

A wireless sensor network is a wireless network consisting of spatially distributed autonomous devices using sensors to cooperatively monitor/control physical or environmental conditions ${ }^{(5)}$. WSN can sense and gather information of various environment or monitoring objects in the monitored area by the network in real time. After processing the information, it delivers to the users by self-group and multi-hop network, for achieving the purpose that monitoring and management of user's physical world. Briefly a WSN can generally be described as a network of nodes that cooperatively sense and control the environment, enabling interaction between human or computers and the surrounding environment ${ }^{(6)}$.

WSN is composed of nodes, which contains radio transceiver, microcontroller and electronic circuits respectively. As shown in Figure 1, the sensor nodes are distributed in the monitoring area, and the sensor nodes constitute a network with different topology. After the initial processing of probing information, the sensor nodes transmits it to the sink node by multi-hop relay, and then the information is transmitted via some methods, such as Satellite, Internet, Mobile communication network, to the end users. End users can also manage and configure the sensor network through management nodes, such as publishing monitoring tasks. 


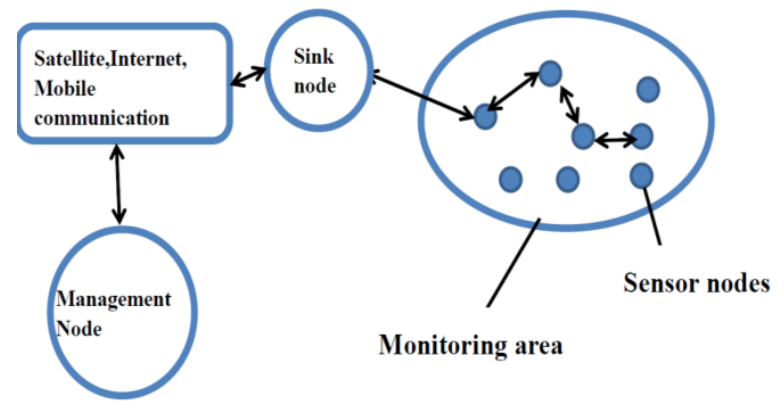

Fig. 1. Schematic Diagram of WSN.

\subsection{Characteristics of WSN}

The several significant characteristics of WSN are described as follows:

1) WSN has large spatial distribution area with huge number nodes, or it can be said that WSN has the scalability to large scale of distribution.

2) The energy of WSN is restricted; as WSN is used to a harsh environment which is harmful to the human body or unattended environment, the consumption of power limits because of nodes powered by the battery, while in some cases, sensors can stay operational for over 10 years on batteries ${ }^{(7)}$, this requires that the network power consumption is small to extend the network life and save power as much as possible.

3) The self-organization of WSN; as the sensor nodes are mostly deployed in the environments like unattended place, WSN has the capability of self-organization.

4) The topology of WSN is dynamic; it needs self-adjustment when network topology changes frequently, such as the damage of the nodes or additional sensor node.

\section{Applications of WSN}

The development of WSNs was inspired by military applications, notably surveillance in conflict zones. Today, they consist of distributed independent devices that use sensors to monitor the physical conditions with their applications extended to industrial infrastructure, automation, health, traffic, and many consumer areas ${ }^{(8)}$. This paper discusses three aspects applications of WSN: industrial applications, smart home, and environment monitoring.

\subsection{Industrial applications}

As the environment of industrial is complex, it integrates workers, equipment and environment. To improve the production efficiency and ensure production safety, abundant information should be collected, such as the temperature of the environment and the status of equipment operation. And some situations which are harmful to humans' health may exist. The industrial wireless sensor networking (WSN) value proposition has evolved from simply extending or replacing wired networks to providing cloud-connected smart object intelligence ${ }^{(9)}$. According to ON World ${ }^{(10)}$, wireless devices to be installed in industrial fields will increase by $553 \%$ between 2011 and 2016 when there will be 24 million wireless-enabled sensors and actuators, or sensing points, deployed worldwide. Among these, $39 \%$ will be used for new applications that are only possible with wireless sensor networking. By 2014, the number of WSN devices will account for $15 \%$ of the entire industrial measurement and control equipment sensing points, and $33 \%$ by 2016 .

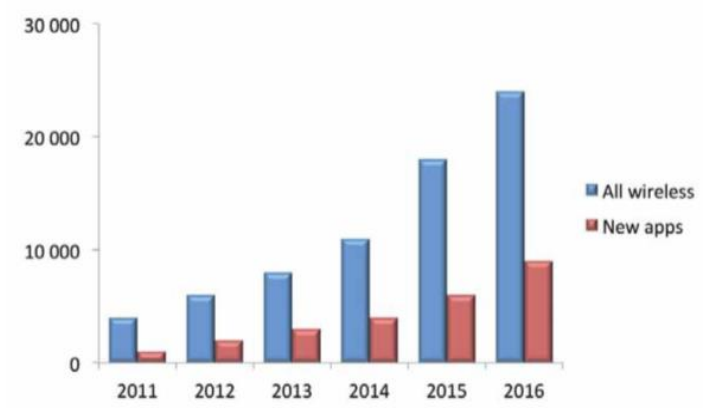

Fig. 2.Global installed industrial wireless sensing points ${ }^{(10)}$.

Here, a simple WSN application for industrial process is illustrated, and this industrial process is related to monitor and control of reactor temperature. Process monitoring and control is a combination of architectures, mechanisms, and algorithms used in the industrial factory for monitoring and control the activities of a specific process to achieve the goal ${ }^{(11)}$. There are four devices: controller, gateway/router, temperature transmitter and value positioner, which are connected with each other by WSN. Its temperature is measured by a temperature sensor, which is used in a special function by the controller to decide how to adjust so that the devices can reach the desired temperature. All those data information is transmitted to the controller by the WSN for using at present or the futur reference.

The advantages of using industrial WSN over industrial wired process include as follows: No wiring constraints; Easy disposition; Easy maintenance; Low cost...

\subsection{Smart home}

A smart home is a space or a room which is provided 
with the ability to get accustomed by itself to certain situations to make the occupants feel comfortable ${ }^{(12)}$. Recently, the smart home has become a global hot topic, and WSN is a significant part of the smart home. In fact, the appearance of smart home can be traced back to the $80 \mathrm{~s}$, while it has even not developed well until now. However, the research of smart home never desists. Nowadays, the intelligent control of family equipment are developed quickly, such as using the wireless sensor network of mobile phone to adjust the light, air conditioning to change the indoor temperature and control the door clock, etc. And some functions of smart home are shown in Figure 3.

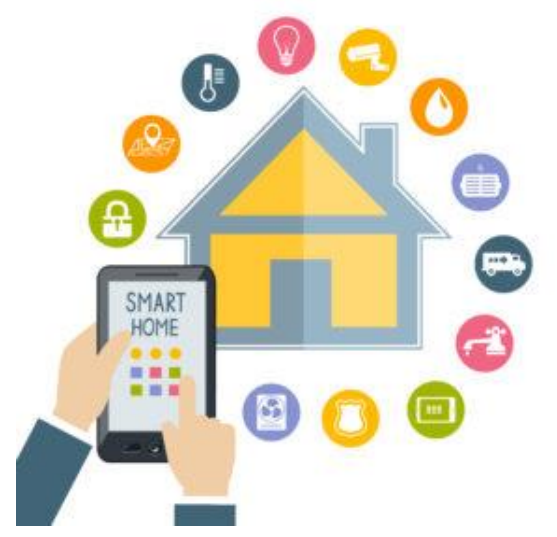

Fig. 3. Smart Home ${ }^{(13)}$

As described in section 2.2, a significant characteristic of WSN is the energy of WSN is restricted. For making the battery more durable, the IEEE 802.15.4 and ZigBee should be mentioned. The importance and application has been increased by the recent delivery of the IEEE 802.15.4 standard ZigBee standard ${ }^{(14)}$, IEEE802.15.4 is a technical standard which defines the operation of low-rate wireless personal area networks (LR-WPANs). ZigBee is a low-power LAN protocol which based on IEEE802.15.4 standard. IEEE 802.15.4 ZigBee offers tremendous specifications to short range and urban environment wireless sensors networks. IEEE 802.15.4 ZigBee standards operate on the license-free industrial, scientific, medical (ISM) frequency band ${ }^{(15)}$.

WSN with ZigBee can provide the low-cost and low-energy consumption for the smart home._Each sensor connects WSN and transmits the information through the gateway or router to the controller. At the same time, the controller controls and adjusts these sensors to fulfill the needs of human.

\subsection{Environmental monitoring}

Environmental monitoring should be the most practical application of WSN, such as forest fire detection, water monitoring, and meteorological monitoring, and so on. All of these use the WSN technology, as the sensors of WSN gather information from these environments and then transmit the information to end users.

Here, the forest fire detection is presented in detail. Many temperature sensors are deployed in the whole forest randomly, and these sensors compose the nodes of this WSN. When the sensor detects the abnormal temperature, it transmits the measurement to the base station, which is a special node for connecting the computer and delivering significant information. A deployment of forest fire detection is shown in Fig 4.

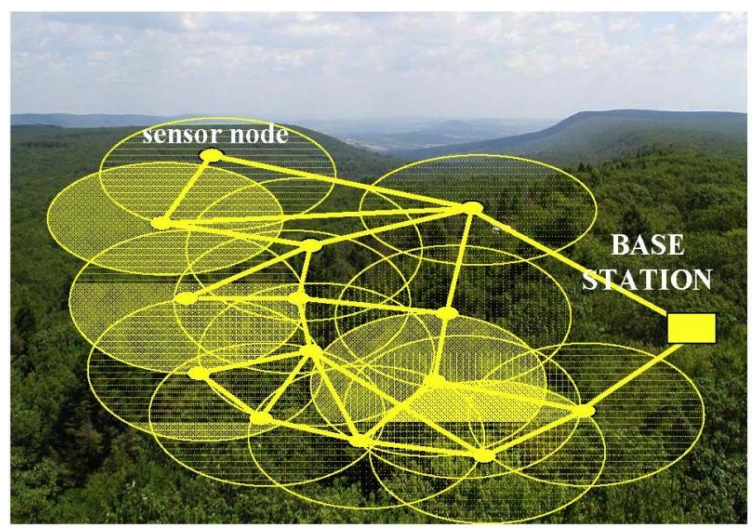

Fig. 4. Forest Fire Detection ${ }^{(16)}$

The vastness of the forest brings a challenge to the protection of forests, the forest fire detection with WSN can overcomes this challenge, it reduces the cost of artificial monitoring, and it is timelier, therefore it is better to prevent natural disasters in advance.

\section{Conclusions}

With the popularity of wireless devices, more and more advantages of WSN are detected by people. In this paper, three applications of WSN were reviewed. Industrial application is more benefit to monitor the unattended area or the space which is harmful to man. WSN is the indispensable part of smart home as it makes smart home more intelligent. In huge area, WSN connect the nodes to detect abnormal phenomenon, and deliver this measurement for preventing disasters. To sum up, the world needs WSN, since it can be applied to different fields and make the life 
more convenient. In the future, there will be more and more fields applying WSN.

\section{References}

(1) K. O'Flaherty, "Securing the internet of things," SC Magazine

$\mathrm{UK}$,

2015. https://www.scmagazineuk.com/securing-the-internet-o f-things/article/541538/ Access date:2017.1.6

(2) Cisco solution, "What Is a Wireless Network?: The Basics",Online:http://www.cisco.com/c/en/us/solutions /small-business/resource-center/work-anywhere/wirele ss-network.html. Access date:2017.1.6

(3) C.Y CHONG and S. P KUMAR, "Sensor networks: Evolution, opportunities, and challenges". Proceedings of the IEEE 91(8), pp. 1247-1256, 2003.

(4) Mauricio Tellez, Samy El-Tawab, and M. Hossain Heydari, "IoT Security Attacks Using Reverse Engineering Methods On WSN Applications" Internet of Things (WF-IoT), 2016 IEEE 3rd World Forum on pp.182-183, 2016.

(5) K. M. M. W. N. B. Narampanawe, M.A.U.S. Navaratne and J. V. Wijayakulasooriya, "Self organizing wireless sensor network with distributed intelligent (SOWDI)" Industrial and Information Systems (ICIIS), 2009 International Conference on, pp.101-102, 2009.

(6) SENSEI. "Integrating the physical with the digital world of the network of the future". http://www.sensei-project.eu/ Access date:2017.1.6

(7) Hwaiyu Geng. "Internet of Things and Data Analytics Handbook" ISBN: 978-1-119-17364-9, pp.301-304, 2016.

(8) Wireless Sensor Networks project team, "Internet of Things: Wireless Sensor Networks", White Paper Publication, p78, 2014.

(9) Mareca Hatler: "Industrial wireless sensor networks: Trends and developments". ON World's 2014, https://www.isa.org/intech/201504web/ Access date:2017.1.6.

(10)M. HATLER, D. GURGANIOUS, and C. CHI, "Industrial wireless sensor networks. A market dynamics report", pp.23-24, 2015.

(11) Gang Zhao, "Wireless Sensor Networks for Industrial Process Monitoring and Control: A Survey", Network Protocols and Algorithms, Vol. 3, 1,pp.47-48, 2011.

(12)Dhiren Tejani, Ali Mohammed and A. H. Al-Kuwari,
"Energy Conservation in Smart Home", 5th IEEE International Conference on Digital Ecosystems and Technologies, Daejeon, Korea,pp. 3-5, May 2011.

(13) Redazione, "Rising interest in IoT and smart home." 21 June 2016 in Technologies. http://www.householdappliancesworld.com/2016/06/21 /rising-interest-iot-smart-home/ Access date:2017.1.6

(14) Nidhi Patel, Hiren Kathiriya. "WIRELESS SENSOR NETWORK USING ZIGBEE". IJRET, Volume: 02 Issue: 06 | Jun-2013, Available @ http://www.ijret.org.

(15) Hemant Ghayvat. "WSN- and IOT-Based Smart Homes and Their Extension to Smart Buildings". Sensors 2015, pp.10350-10379,2015.

(16) S. Del Favero, "Networked Control Systems". http://automatica.dei.unipd.it/research/networked-contr ol-systems.html Access date:2017.1.6 